\title{
Modified Split-Step Fourier Method for Compensation of Nonlinear Fibre Impairments
}

\author{
Danish Rafique*, Marco Mussolin**, Marco Forzati***, \\ Jonas Mårtensson***, Mohsan N. Chugtai***, Andrew D. Ellis* \\ * Photonics Systems Group, Tyndall National Institute and Department of Electrical Engineering/Physics, \\ University College Cork, Dyke Parade, Prospect Row, Cork, Ireland \\ ${ }^{* *}$ Department of Information Engineering, University of Padova, Italy \\ ${ }^{* * *}$ Acreo Netlab, Electrum 236, SE 16440, Kista, Sweden \\ Tel: 00(353) 490 4866, email: danish.rafique@,tyndall.ie
}

\begin{abstract}
In this paper, we investigate a modified split-step Fourier method to enable computationally-efficient digital nonlinearity compensation for a coherently-detected $112 \mathrm{~Gb} / \mathrm{s}$ polarization multiplexed quadrature phase shifted keying transmission over a $1,600 \mathrm{~km}$ link $(20 \times 80 \mathrm{~km})$ with no inline compensation. We report up to $80 \%$ reduction in required stages to perform nonlinear compensation, in comparison to the conventional backpropagation algorithm. This method takes into account the correlation between adjacent symbols at a given instant using a weighted-average approach to enable practical digital nonlinearity compensation.
\end{abstract}

Keywords: Coherent communications, Kerr nonlinearity, digital signal processing, advanced modulation formats, nonlinear optics

\section{INTRODUCTION}

The optical coherent receiver with digital signal processing (DSP) is a promising technology for next generation $100 \mathrm{~Gb} / \mathrm{s}$ transmission systems because it can offer higher optical signal-to-noise ratio (OSNR) tolerance and compensation of linear fibre impairments $[1,2]$. Despite such benefits, practical $100 \mathrm{~Gb} / \mathrm{s}$ transmission systems may require further performance improvements in order to achieve similar reaches as current systems operating at $10 \mathrm{~Gb} / \mathrm{s}$. One possible approach to achieve this goal is to increase the optical power and thus received OSNR while maintaining the nonlinear impairments as low as possible with a help of DSP [3, 4]. With the availability of advanced DSP techniques and high-speed analogue-to-digital converters for 40- and $100 \mathrm{~Gb} / \mathrm{s}$ systems, electronic mitigation of transmission impairments has matured over past few years. In particular, electronic signal processing using digital back-propagation (DBP) with inverse fibre parameters or time inversion has been applied to the compensation of channel nonlinearities [5-7]. However, the complexity of DBP is currently exorbitant due to significantly high number of processing steps required in such calculations. In order to address these issues, simplifications in the DBP algorithm employing single-step per span or less via DBP techniques [810] have already commenced.

In this paper we investigate a simplified DBP algorithm based on the correlation of signal power in neighbouring symbols when applying nonlinear phase compensation which requires less than one processing step per transmission span. We test the algorithm on a $112 \mathrm{~Gb} / \mathrm{s}$ polarization multiplexed quadrature phase shifted keying (PM-QPSK) system, in a $1,600 \mathrm{~km}(20 \times 80 \mathrm{~km})$ transmission system, and show that a considerable complexity reduction (in terms of reduced back-propagation stages) can be achieved compared to standard DBP methods [8].

\section{THEORY (WEIGHTED DIGITAL BACK-PROPAGATION)}

Digital back-propagation processes the received signals by launching them into a virtual fibre with link parameters of opposite-sign values of those in the transmission channel. In practice, this method can be implemented by calculating the nonlinear Schrödinger equation (NLSE) governing the signal propagation through the fibre channel at the receiver. Details of this method can be found in $[5,6]$.

The principle of weighted DBP (WDBP) algorithm is to correlate the nonlinear shift at a specific symbol location with the power of various consecutive symbols, rather than only one, thus taking into account the phenomenon of dispersion induced power "spilling" into neighbouring symbols. Indeed, this causes the power profile at each amplifier stage (i.e. when power is highest and most of the nonlinear shift takes place) to be the overlap of a larger number of dispersion-broadened pulses. Fig. 1a shows the schematic of the WDBP method, similar in concept to the asymmetric SSFM [5] approximation to solve the NLSE, albeit with a much coarser step-size. The algorithm consists of a series of linear and nonlinear elements, where each LC is responsible for CD compensation using FFT/IFFT algorithms [11] and each NLC is responsible to compensate nonlinear phase shift imposed along a certain fibre section. Fig. 1b shows the schematic of the modified nonlinear segment. The incoming digital data streams correspond to complex electric field vectors for $x$ and $y$ polarization states, and the correlation between neighbouring symbols is taken into account by applying a time-domain filter corresponding 
to the weighted sum of neighbouring symbols. Given a coarse step-size ( $>$ dispersion length), nonlinear phase shift (NLPS) on a given symbol is a weighted NLPS from adjacent symbols, as shown in equation (1a) and equation (1b),

$$
\begin{aligned}
& E_{x}{ }^{\text {out }}=E_{x}^{\text {in }} \times \exp \left[-j \sum_{k=-(N-1) / 2}^{(N-1) / 2} c_{k}\left\{a\left|E_{x}^{\text {in }}\left(t-k \frac{T_{s}}{2}\right)\right|^{2}+b\left|E_{y}^{i n}\left(t-k \frac{T_{s}}{2}\right)\right|^{2}\right\}\right] \\
& E_{y}{ }^{\text {out }}=E_{y}{ }^{\text {in }} \times \exp \left[-j \sum_{k=-(N-1) / 2}^{(N-1) / 2} c_{k}\left\{a\left|E_{y}^{\text {in }}\left(t-k \frac{T_{s}}{2}\right)\right|^{2}+b\left|E_{x}^{\text {in }}\left(t-k \frac{T_{s}}{2}\right)\right|^{2}\right\}\right]
\end{aligned}
$$

where $E_{x, y}{ }^{\text {in }}$ and $E_{x, y}{ }^{\text {out }}$ are the electric fields for orthogonal polarization states before and after WDBP for $x$ and $y$ polarization states, $a$ and $b$ represent intra-polarization and inter-polarization parameters [8], $N$ represents the number of symbols (or filter length) to be considered for a nonlinear phase shift, $c_{k}$ is the weighing vector, $k$ is the delay order, and $T_{s}$ is the symbol period. Note that the values for a and $b$ were found to be almost identical since we considered the Manakov model [12] for fibre propagation; and further details on optimization of these parameters can be found in [8]. It also is worth mentioning that the complexity reduction via WDBP is primarily achieved from the reduction in required FFTs rather than the reduced number of steps for NLC calculations.

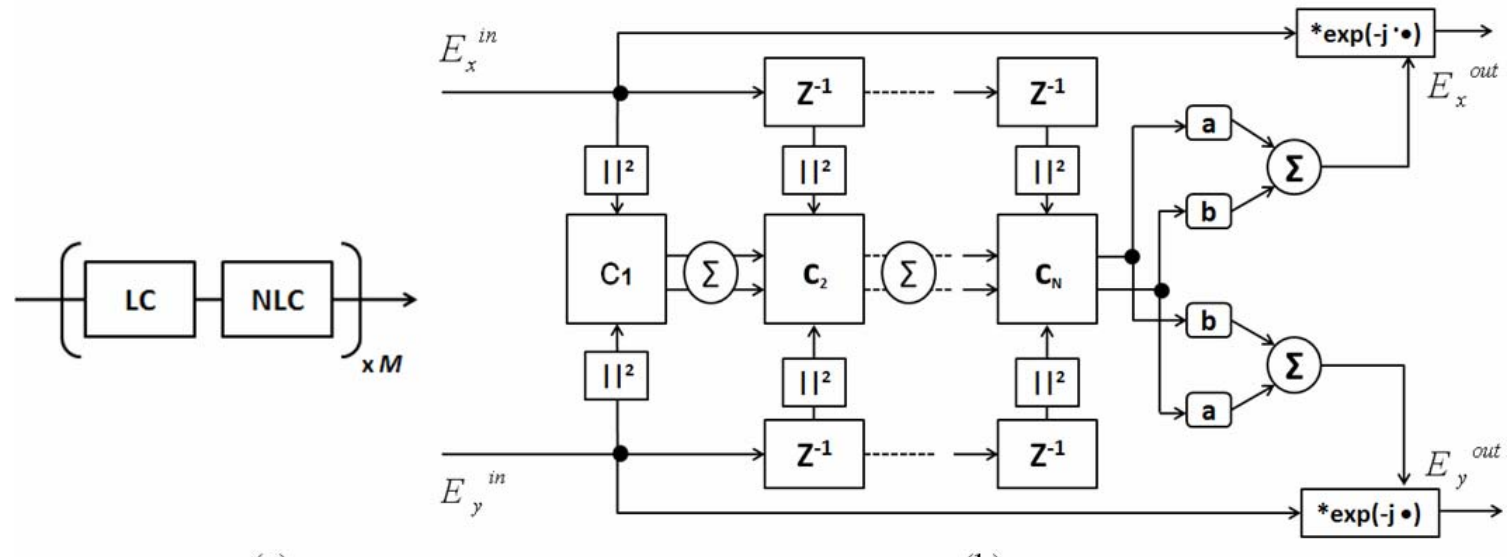

(a)

(b)

Figure 1: a) Schematic of weighted digital back-propagation method, b) Schematic of modified nonlinear compensation segment.

\section{SIMULATION MODEL}

Figure 2 depicts the PM-QPSK transmitter setup at $112 \mathrm{~Gb} / \mathrm{s}$, the transmission link, and the receiver structure, similar to [7]. The transmitter consists of a $1550 \mathrm{~nm}$ laser with $500 \mathrm{kHz}$ line-width, followed by a polarization beam splitter (PBS), and two nested Mach Zehnder modulators (one per polarization component), each driven by two $28 \mathrm{~Gb} / \mathrm{s}$ bit sequences of length $2^{n}$ which were properly pre-coded in order for the resulting QPSK optical signal to be phase-modulated by a De Bruijn binary sequence (DBBS,) $\mathrm{B}(4, n)$, of order $n$. Different sequence orders ( $n=13$, and $n=12$ respectively) were used in order to obtain un-correlated data for each polarization component. Finally the two polarization components were combined by means of an ideal polarization beam combiner and the resulting $112 \mathrm{~Gb} / \mathrm{s}$ PM-QPSK was passed through a $35 \mathrm{GHz} 3^{\text {rd }}$ order Gaussian filter and sent over the transmission link consisting of twenty spans comprising an erbium doped fibre amplifier (EDFA) followed by $80 \mathrm{~km}$ of standard single mode fibre (SSMF). The fibre had attenuation $(\alpha)$ of $0.2 \mathrm{~dB} / \mathrm{km}$, dispersion (D) of $16 \mathrm{ps} / \mathrm{nm} / \mathrm{km}$, and a nonlinearity coefficient $(\gamma)$ of $1.5 / \mathrm{W} . \mathrm{km}$. The optical amplifiers were modelled as ideal noise-free EDFAs and white Gaussian noise was added at the receiver in order to calculate the OSNR required to obtain $\mathrm{BER}=10^{-3}\left(\mathrm{OSNR}_{\mathrm{req}}\right)$. Cross-polarization non-linear effects were included according to the Manakov model [12] and polarization mode dispersion (PMD) was neglected.

After fibre transmission, the received signal was pre-amplified, filtered using a $35 \mathrm{GHz}$ bandwidth $3^{\text {rd }}$ order Gaussian optical band-pass filter. The optical signal was then coherently detected using a homodyne receiver, low pass filtered and down-sampled to 2 samples per symbol. The digital field was reconstructed using the inphase/quadrature components of each polarization and the signal was digitally processed. The transmission impairments were compensated using our WDBP algorithm explained in the previous section, where a series of $L C+N L C$ steps was employed and the coefficients $a, b$ and $N$ were optimized. After the compensation of CD and non-linear phase shift, polarization demultiplexing was performed using a standard butterfly structure using four CMA adaptive filters with up to 7 taps each (the number of filter taps were optimized for each OSNR value) and the carrier phase was recovered using the Viterbi and Viterbi method. Finally the symbol decisions were 
made and errors were counted. All the numerical simulations were carried out using VPI@V.8.3, and digital postprocessing was performed in Matlab®v.7.10.

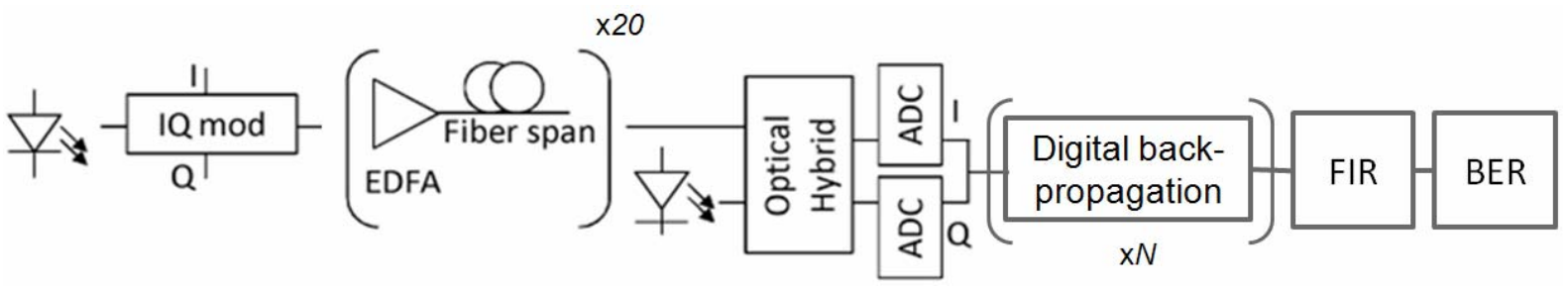

Figure 2. Simulation setup for $112 \mathrm{~Gb} / \mathrm{s}$ PM-QPSK with 20 spans employing digital back-propagation with $N$ steps for the whole link. IQ: in-phase and quadrature, FIR: Finite impulse response, ADC: Analogue to digital converter.

\section{RESULTS AND DISCUSSIONS}

In order to establish the optimum time-domain filter profile (shape and filter coefficients) for WDBP algorithm, we employed various time-domain filters, and identified Gaussian approximation to perform the best (lowest BER), and consequently the filter profile was fixed to be Gaussian in the following analysis. Note that the minimum number of required taps for nonlinear stage with the step-sizes of $1,2,4$, and 10 were found to be 21 , 17,15 and 7 , respectively.

Figure 3 a depicts the performance of the WDBP algorithm with varying precision (measured as total number of back-propagation steps). The results are plotted in terms of $\mathrm{OSNR}_{\text {req }}$ as a function of launch power $\left(P_{i n}\right)$. In the absence of nonlinear compensation, $\mathrm{OSNR}_{\text {req }}$ degrades rapidly and above $4 \mathrm{dBm}$, BER of $10^{-3}$ cannot be achieved due to strong intra-channel nonlinear effects at high launch powers. However, when WDBP is employed with only single step for the whole link, a significant improvement of about $\sim 2.5 \mathrm{~dB}$ is observed, e.g. at $4 \mathrm{dBm}$. As the precision of WDBP is enhanced, one can see a gradual improvement in $\mathrm{OSNR}_{\text {req }}$, e.g. $\sim 4.5 \mathrm{~dB}$ OSNR $_{\text {req }}$ improvement with respect to the case with no NLC can be observed with 20 steps at the $\mathrm{P}_{\text {in }}$ of $4 \mathrm{dBm}$. Nevertheless, there is still a visible penalty with respect to the back-to-back OSNR ${ }_{\text {req }}$, due to the coarse step-size employed to keep the step-count minimal. We expect the performance to improve further given higher number of steps per span are employed, as it has been reported previously [7].

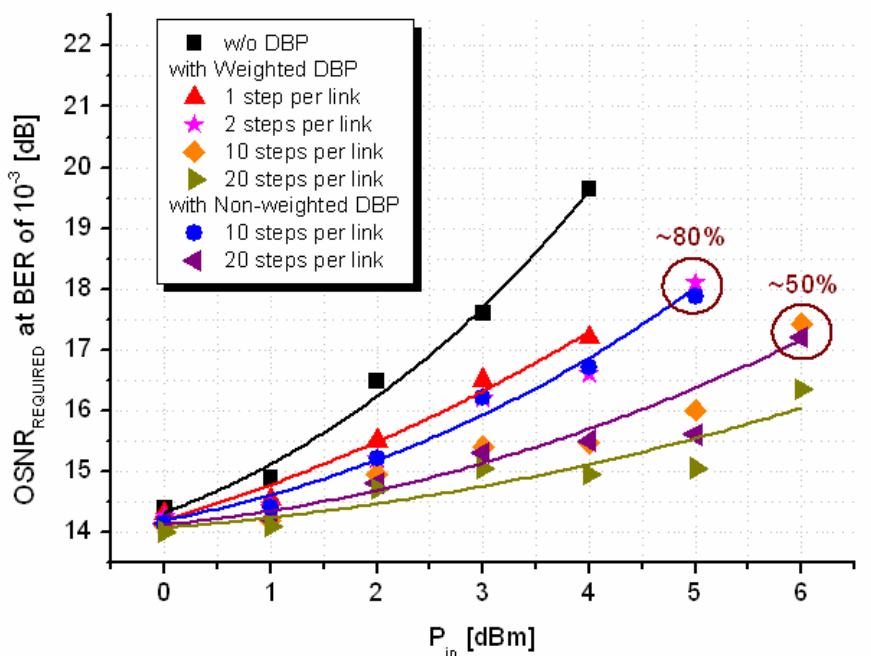

(a)

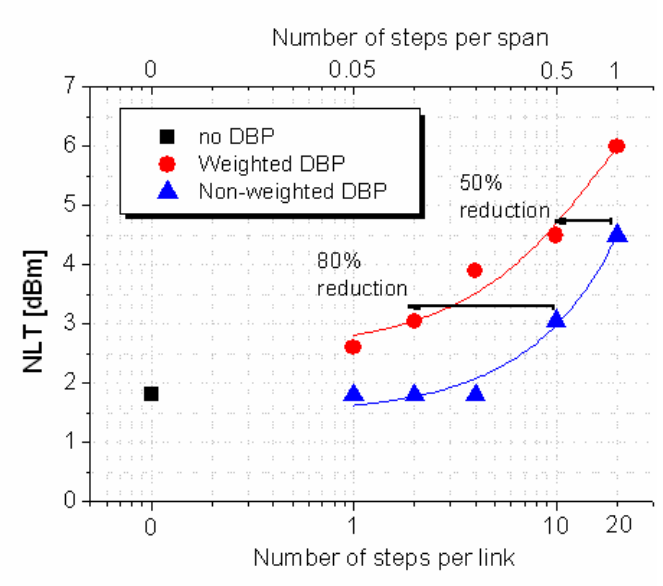

(b)

Figure 3: a) Required OSNR as a function of launch power after 20 spans. No DBP (squares), WDBP: 1 step (up triangles), 2 steps (stars), 10 steps (diamonds), NWDBP: 10 steps (circles), 1 step (left triangles), b) Nonlinear threshold defined at $2 \mathrm{~dB} O \mathrm{OSNR}_{\text {req }}$ penalty with respect to back-to-back case for various cases in Fig. $3 a$. No DBP (square), WDBP (circles), NWDBP (triangles).

In order to compare our results with previously proposed DBP methods, we also employed recently proposed asymmetric SSFM based DBP [8], we will call it non-weighted DBP (NWDBP), which is based on instantaneous power dependent nonlinear phase shift and employs single step per span for DBP calculations. Comparing the two approaches, it can be observed that the OSNR req with 10 steps for the whole link (NWDBP) is equivalent to that of WDBP algorithm with only 2 steps for the link. This shows a significant $80 \%$ reduction in required DBP steps with our new approach. Similarly, as we increase the number of step with WDBP method, the performance for 10 steps WDBP and 20 steps NWDBP almost converge, i.e. $\sim 50 \%$ less step calculations. 
Note the decrease in the margin of complexity reduction as the number of steps is increased for WDBP method. This is due to the better precision for NWDBP when higher number of steps are employed, leaving less scope of improvement with WDBP, however one can still observe that 20 steps for the whole link with WDBP method still outperforms the NWDBP algorithm. This trend is also shown in Fig. $3 \mathrm{~b}$ which shows the nonlinear threshold (NLT) - defined as the launch power for which the $\mathrm{OSNR}_{\text {req }}$ is increased by $2 \mathrm{~dB}$ compared to back-toback - for all the cases in Fig. 3a. Here one can clearly see that the nonlinear tolerance of WDBP is significantly better than traditional DBP method and one can employ as few as 2 steps for the whole link. It is worth mentioning that complexity reductions (reduced number of required DBP steps) in proportion to the ones' reported here, have been recently proposed using a back-propagation technique employing an analogous frequency-domain filtering approach [9], which further validates our results.

\section{CONCLUSIONS}

We have shown that the required number of digital back-propagation stages can be reduced significantly by considering the correlation between adjacent symbols. We report a significant reduction in required backpropagation steps, up to $80 \%$ compared to the previously proposed conventional DBP methods, for $112 \mathrm{~Gb} / \mathrm{s}$ PM-QPSK system after $1,600 \mathrm{~km}(20 \mathrm{x} 80 \mathrm{~km})$. In view of near future optical network deployments we believe that this approach will make nonlinear compensation practically viable, given the radical complexity reductions that have been reported.

\section{ACKNOWLEDGEMENTS}

The work described in this paper was carried out with the support of the EUROFOS project, Network of Excellence funded by the European Commission through the $7^{\text {th }}$ ICT-Framework Programme; Science Foundation Ireland under Grant 06/IN/I969p; the Italian Ministry of University and Research project PRIN 2008MPSSNX, the Italian Ministry of Foreign Affairs (Direzione Generale per la Promozione e la Cooperazione Culturale) and the agreement with ISCTI, Rome, Italy.

\section{REFERENCES}

[1] C. S. Fludger, T. Duthel, D. vanden Borne, C. Schulien, E.-D. Schmidt, T. Wuth, J. Geyer, E. DeMan, G.-D. Khoe, and H. de Waardt, "Coherent equalization and POLMUX-RZ-DQPSK for robust 100-GE transmission," J. Lightwave Technol. 26, 64 (2008).

[2] G. Charlet, J. Renaudier, H. Mardoyan, P. Tran, O.B. Pardo, F. Verluise, M. Achouche, A. Boutin, F. Blache, J.Y Dupuy, and S. Bigo: Transmission of 16.4-bit/s capacity over $2550 \mathrm{~km}$ using PDM QPSK modulation format and coherent receiver, J. Lightwave Technol. 27, 153, 2009.

[3] M. Kuschnerov, F. N. Hauske, K. Piyawanno, B. Spinnler, M. S. Alfiad, A. Napoli, and B. Lankl: DSP for coherent single-carrier receivers, J. Lightwave Technol. 27, 3614, 2009.

[4] K. Kikuchi: Electronic post-compensation for nonlinear phase fluctuations in a 1000-km 20-Gbit/s optical quadrature phase-shift keying transmission system using the digital coherent receiver, Opt. Express 16, 889, 2008.

[5] E. Ip: Nonlinear compensation using backpropagation for polarization-multiplexed transmission, J. Lightwave Technol. 28, 939, 2010.

[6] X. Li, X. Chen, G. Goldfarb, Eduardo Mateo, I. Kim, F. Yaman, and G. Li: Electronic post-compensation of WDM transmission impairments using coherent detection and digital signal processing, Opt. Express 16, 880, 2008.

[7] D. Rafique, J. Zhao, and A.D. Ellis, "Digital back-propagation for spectrally efficient WDM 112 Gbit/s PM M-ary QAM transmission," Opt. Express 19, 5219-5224, 2011.

[8] S. Oda, T. Tanimura, T. Hoshida, C. Ohshima, H. Nakashima, T. Zhenning, and J.C. Rasmussen: 112 Gb/s DP-QPSK transmission using a novel nonlinear compensator in digital coherent receiver, in Proc. Optical Fiber Communication Conference'09, OThR6, 2009.

[9] L. B. Du and A. J. Lowery: Improved single channel backpropagation for intra-channel fiber nonlinearity compensation in long-haul optical communication systems, Opt. Express 18, 17075, 2010.

[10] X. Chongjin and R.J. Essiambre: Electronic nonlinearity compensation in 112-Gb/s PDM-QPSK optical coherent transmission systems, in Proc. European Conference on Optical Communications ECOC'10, Mo.1.C.1, 2010.

[11] S.J. Savory, G. Gavioli, R.I. Killey, and P. Bayvel: Electronic compensation of chromatic dispersion using a digital coherent receiver, Opt. Express, 15, 2120, 2007.

[12] D. Marcuse, C. R. Manyuk, and P. K. A. Wai: Application of the Manakov-PMD equation to studies of signal propagation in optical fibers with randomly varying birefringence, J. Lightwave Technol. 15, 17351746, 1997. 